\title{
BMJ Open Patient and provider perspectives on barriers to screening for diabetic retinopathy: an exploratory study from southern India
}

\author{
Shuba Kumar, ${ }^{1}$ Geetha Kumar, ${ }^{2}$ Saranya Velu, ${ }^{2}$ Shahina Pardhan, ${ }^{3}$ \\ Sobha Sivaprasad, ${ }^{4}$ Paisan Ruamviboonsuk, ${ }^{5}$ Rajiv Raman (1) ${ }^{2}$
}

To cite: Kumar S, Kumar G, Velu S, et al. Patient and provider perspectives on barriers to screening for diabetic retinopathy: an exploratory study from southern India. BMJ Open 2020;10:e037277. doi:10.1136/ bmjopen-2020-037277

- Prepublication history and additional material for this paper are available online. To view these files, please visit the journal online (http://dx.doi org/10.1136/bmjopen-2020037277).

Received 04 February 2020 Revised 05 November 2020 Accepted 10 November 2020

\section{Check for updates}

(c) Author(s) (or their employer(s)) 2020. Re-use permitted under CC BY-NC. No commercial re-use. See rights and permissions. Published by BMJ.

${ }^{1}$ Samarth, Chennai, Tamil Nadu, India

${ }^{2}$ Shri Bhagwan Mahavir

Vitreoretinal Services, Sankara Nethralaya, Chennai, India

${ }^{3}$ Vision and Eye Research Unit (VERU), School of Medicine, Anglia Ruskin University,

Cambridge, UK

${ }^{4}$ NIHR Moorfields Biomedical Research Centre, Moorfields Eye Hospital NHS Foundation Trust, London, UK

${ }^{5}$ Department of Ophthalmology, Rajavithi Hospital, Bangkok, Thailand

Correspondence to Dr Rajiv Raman rajivpgraman@gmail.com

\section{ABSTRACT}

Objective Diabetic retinopathy is one of the leading causes of visual impairment after cataract and uncorrected refractive error. It has major public health implications globally, especially in countries such as India where the prevalence of diabetes is high. With timely screening and intervention, the disease progression to blindness can be prevented, but several barriers exist. As compliance to diabetic retinopathy screening in people with diabetes is very poor in India, this study was conducted to explore understanding of and barriers to diabetic retinopathy screening from the perspectives of patients and healthcare providers.

Methods Using qualitative methods, 15 consenting adult patients with diabetes were selected purposively from those attending a large tertiary care private eye hospital in southern India. Eight semistructured interviews were carried out with healthcare providers working in large private hospitals. All interviews were audiotaped, transcribed verbatim and analysed using the framework analytical approach.

Results Four themes that best explained the data were recognising and living with diabetes, care-seeking practices, awareness about diabetic retinopathy and barriers to diabetic retinopathy screening. Findings showed that patients were aware of diabetes but understanding of diabetic retinopathy and its complications was poor. Absence of symptoms, difficulties in doctor-patient interactions and tedious nature of follow-up care were some major deterrents to care seeking reported by patients. Difficulties in communicating information about diabetic retinopathy to less literate patients, heavy work pressure and silent progression of the disease were major barriers to patients coming for follow-up care as reported by healthcare providers.

Conclusions Enhancing patient understanding through friendly doctor-patient interactions will promote trust in the doctor. The use of an integrated treatment approach including education by counsellors, setting up of patient support groups, telescreening approaches and use of conversation maps may prove more effective in the long run.

\section{INTRODUCTION}

Diabetic retinopathy (DR), a microvascular complication in the eye due to uncontrolled
Strengths and limitations of this study

- This was a qualitative study that explored barriers to diabetic retinopathy screening from the perspectives of patients and healthcare providers (HCP) which enabled a more comprehensive understanding of the phenomenon.

- Insights obtained from patients and providers have given good cues for development of intervention strategies.

- The study could have benefited from interviews with family members, who play an important role both in decision-making for care seeking and in providing support to patients.

- A larger patient sample representing a wider patient demographic could have provided wider perspectives.

- Inclusion of HCPs from smaller eye clinics would have provided additional perspectives further enhancing understanding of the phenomena.

diabetes, has high prevalence in Africa $(33.8 \%)$ and in the Western Pacific $(36.2 \%){ }^{1}$ In another study, the highest age standardised prevalence was among Caucasians at $45.8 \%$ with Asians (combined) at $19.9 \% .^{2}$ Flaxman et $a l^{3}$ in their systematic review reported that blindness due to DR has been on the rise from 1990 until 2015. In India, the disease has major public health implications due to two main reasons: (1) an estimated 57 million people will have diabetes by $2025(195 \%$ increase from 1995) and (2) the risk of sightthreatening retinopathy is higher in adults with diabetes. ${ }^{4}$ Previous population-based studies from India have reported prevalence of DR to be $10 \%$ in rural areas and $18 \%$ in urban areas. ${ }^{5}$ Moreover, sight-threatening DR affects $5 \%$ of people with diabetes, that is, 4.5 million, which is stated to increase as the number of people with diabetes increases. ${ }^{6}$

The management of diabetic eye disease in India (online supplemental file 1) is 
influenced by a lack of screening programmes, poor public awareness on diabetic eye disease and poor understanding of the need for regular retinal screening. ${ }^{7}$ Most retinal services in India that manage these patients are not publicly funded. There is also a wide variation in provision of healthcare ranging from highly specialised hospitals to basic facilities without trained ophthalmologists.

Shukla $e t$ al assessed the perceptions of care and challenges faced in availing care among people with diabetes in India and reported that $45 \%$ of participants already had vision loss when they first presented to an eye facility and before their DR was even detected. ${ }^{8}$ Lingam et al in their study on the uptake of DR screening in a pyramidal model of eye healthcare found that $2 \%$ at tertiary level, $40 \%$ at secondary and $50 \%$ at primary level had never undergone previous dilated eye examination. ${ }^{9}$ Given that $50 \%-70 \%$ of DR-related visual impairments can be prevented by timely screening and intervention, ${ }^{10}$ the importance of early identification and regular follow-up cannot be overemphasised. Thus, while DR is one of the leading causes of blindness, vision loss is largely preventable through regular screening and follow-up which continues to be quite inadequate as suggested by previous research. ${ }^{11-14}$

Several barriers identified to screening for DR ranged from financial burden, lack of awareness about the importance of screening, transportation, language barriers, cultural myths, denial, fear and depression. ${ }^{15}$ Piyasena et al found that inter-related user, family and institutional factors influenced the uptake of DR screening and follow-up services in the Western Province of Sri Lanka. ${ }^{16}$ Factors such as older age and physical disability have also been found to act as barriers to screening. A study from India highlighted several issues, which included travelling long distances to access the health facility and cost of travel. ${ }^{8}$ Patient's belief that their eyes were healthy, not having anybody to accompany them to healthcare facilities and financial costs of seeking care were among other barriers reported. ${ }^{17}$ However, most of these findings are from quantitative study ${ }^{15}$ that by their very design are limited in terms of their ability to probe, explore and gain deeper insights. Furthermore, these barriers may be influenced by regional variations. There is thus a paucity of qualitative studies on this topic in India which provided the impetus for this study involving semistructured interviews (SSIs) with both patients and healthcare providers (HCPs).

We included HCPs for two reasons: (1) being care providers their perceptions and experiences would enable a more holistic understanding of this issue, (2) given that HCPs are deeply respected in our culture they could exert a significant role in encouraging patients to get their eyes screened thereby playing an important role in future interventions. From patients we explored their experiences of living with diabetes, how they coped with their condition in terms of care-seeking behaviours as well as lifestyle modifications, their awareness about DR and their perceptions on barriers towards DR screening.
From HCPs, we explored their perceptions on patient understanding of diabetes and DR, the nature of information about diabetes and DR provided to patients and what they believed were barriers for accessing DR care. Getting to understand both points of view helped to build deeper understanding of the phenomenon.

\section{METHODS}

The study was carried out in a tertiary eye care centre run by a non-government organisation (NGO) located in Chennai, capital of the state of Tamil Nadu in South India.

\section{Sampling}

Adult patients with type 2 diabetes mellitus (DM) aged 50 years and above were considered, because only after a few years of living with DM do patients tend to develop DR. The strongest predictor for DR is the duration of diabetes, ${ }^{18}$ therefore patients who had been living with DM for a period of 5 years or more were purposively selected to participate in SSIs. Patients already diagnosed with DR were not included as the emphasis was on awareness about DR, need for eye screening and barriers to screening. Given that 12 interviews are sufficient to reach saturation if the objectives are fairly narrow and the sample not too diverse ${ }^{19}$ and keeping in mind feasibility, logistics and the fact that qualitative research is time consuming, we decided on carrying out 15 interviews with patients. We believed this would be adequate to achieve saturation. Using maximum variation sampling we recruited eight men and seven women of different ages during the period from February to June 2019. The hospital maintains a computerised schedule of patient appointments with various eye specialists inclusive of names of patients, their gender and age. The other details such as education levels and nature of occupation were gathered during the interview. As our focus was on barriers to DR care we reviewed the appointment schedules of the retinal specialists. On the specified dates of the appointments our research team (GK and SV both trained in qualitative research methods by SK) met with patients aged 50 years and above, following their consult and ascertained eligibility. Those eligible were consented to participate in an SSI. The eight HCPs recruited had 5 or more years of experience working with persons with diabetes. Five ophthalmologists were recruited, three worked at the NGO eye hospital and two were from another private eye hospital. The remaining HCPs included two diabetologists and one dietician recruited from a diabetes specialty centre.

Separate open-ended interview guides (online supplemental file 2) for patients and HCPs, informed by literature and our prior interactions with patients, were developed. Broadly, they elicited information on patient's understanding of diabetes, perceptions on their experiences and risks of living with it, lifestyle modifications made, care-seeking behaviours, understanding of DR, barriers 
to DR screening and its importance and suggestions on what would be helpful. The participants were escorted to a quiet area in the hospital where the interviews were carried out. For most patients the interviews were done in Tamil, the language of communication in our state, while with most HCPs it was in English. Informed consent was obtained from all participants following which interviews were conducted and audio recorded. The duration of interviews varied from about 35 to $50 \mathrm{~min}$. All interviews were transcribed verbatim; those in Tamil were translated into English for the purpose of analysis. Every transcript was rechecked with the audio recording by the team to ensure fidelity to the original audiotaped interviews before analysis. All data were anonymised to maintain confidentiality.

\section{Patient and public involvement}

Patients and the public were not involved in the design or conduct of our study.

\section{ANALYSIS}

Analysis followed the framework analytical approach, ${ }^{20}$ which is very suitable for data gathered through SSIs ${ }^{21}$ and began by gaining familiarity with each of the transcripts through repeated readings. We carried out a systematic method of organising our data into spreadsheets, keeping in mind our research questions and listed out several categories like 'understanding of diabetes', 'careseeking practices', 'awareness about DR', 'barriers to DR screening', and so on. We then began extracting relevant portions of text from each interview related to these categories and went through a process of indexing or sifting through the data; sorting and selecting quotes and placing them under the appropriate categories. Developing and refining our categories in this manner helped us to compare and contrast them and determine the ones that could be meaningfully combined and those that were stand-alone thereby setting the stage for theme development. In developing themes, we looked for patterns and made decisions on what themes best explained our data and provided important insights.

\section{FINDINGS}

\section{Patient and HCP characteristics}

All 15 patients were married and their average age was $63.2 \pm 9.2$ years. All of them were living with diabetes

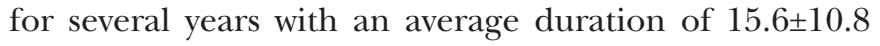
years and had not received any treatment for diabetic eye disease. The eight HCPs, who participated in the interviews, comprised five women and three men. Their average age was $44.7 \pm 8.1$ years and average duration of years of experience was $17 \pm 10.5$ years (online supplemental table 1).

\section{Themes of analysis}

The four themes that best explained the data and addressed our research questions were (1) recognising and living with diabetes, (2) care-seeking practices, (3) awareness about DR, and (4) barriers to DR screening. These were explored from the perspectives of both patients and providers. However, the last two themes have been combined and presented for the HCPs so as to succinctly reflect the manner in which they best described the themes.

\section{Patient perspectives}

Recognising and living with diabetes

Recognition of the fact that they might have diabetes came rather slowly to most patients. For the most part, the diagnosis of diabetes came as a surprise and a great shock. It often started with minor symptoms like a tingling feeling in the extremities, frequent urination, itching sensation while passing urine and feeling unusually thirsty or hungry. These were initially ignored until other symptoms started showing up like loss of weight, feeling faint and dizzy or a wound that was not healing. Most patients did not even suspect that they had diabetes and it was only after they were asked to undergo blood sugar tests on the instruction of the doctor did they come to learn of their diagnosis. Others spoke of not experiencing any symptoms at all and learnt of their condition when they underwent a routine health check-up. A female participant came to know of her diabetes when she underwent surgery for removal of a tumour. Myths surrounding the disease also emerged with one participant stating that he believed he would not get the disease as he thought it only affected the first-born son in the family. The realisation that this was a lifelong condition that could seriously spiral out of control if not carefully managed had begun to dawn on them. A few participants, apart from highlighting their own concerns and worries, were also distressed by the stress and burden their illness would impose on their family members. These were all typically their first reactions to the diagnosis. But with time, regular medication and care provided at their health facilities their understanding of the disease improved as they came to terms with their disease. Some even took on a more proactive role by encouraging others who had the disease to be compliant while others appeared more fatalistic in accepting their situation. Some were more familiar with the disease as their parents, siblings or close relatives were living with it and consequently were emotionally better prepared when told of their diagnosis.

In terms of their understanding of diabetes, most participants were aware that poor control of their blood sugar level could result in a host of health problems and complications. Signs and symptoms ranging from becoming tired easily, losing weight, finding it difficult to work, feeling faint and dizzy to more serious conditions such as kidneys and liver being affected, severe pain in the feet, suffering a stroke or a heart attack were reported. It is important to note that those who had a parent or sibling living with diabetes reported being attuned to developing symptoms at some point and accepted the inevitability of acquiring the disease on account of its 
genetic nature. They were also more aware of the consequences of improper management and spoke of the risks to their health in terms of developing a stroke. The fact that diabetes could impair vision leading to possible loss of sight was also reported by many participants.

The main source of information about diabetes came from their HCPs including doctors and nurses. A few others learnt more about the disease from books, articles and literature on the internet as well as from health programmes on television. They felt that doctors were not too forthcoming and usually did not spend time explaining in detail. Friends, neighbours and family members also served as another information source, more so if they were already diagnosed with diabetes.

\section{Care-seeking practices}

Although many patients never thought to seek care when symptoms initially started, once diagnosed they became more alert to the need to seek regular healthcare. Based on the advice given by their doctors, they started attending clinics to get their blood sugar checked. One female participant spoke of feeling depressed each time she underwent a blood sugar test as the test brought home to her the fact that she had diabetes and had to somehow 'survive with the disease'. While participants appreciated the necessity of these periodic visits to test their blood sugars, they nevertheless found them to be tedious. Therefore, recommendations by doctors to undergo further tests like an eye test, for example, were seen as an added burden both in time and cost and were often resisted. A few participants emphasised the importance of consistently seeing the same doctor so as to avoid unnecessary confusion from varying recommendations. In this context, the manner in which doctors communicated to patients influenced the level of trust and how well patients would comply with their advice. Participants believed that doctors needed to speak gently and not frighten them with harsh consequences which would only result in them going to another doctor. While they agreed that all necessary information needed to be communicated, this needed to be done in a friendly and nonthreatening manner so as to instil confidence.

Use of alternate medicines like Ayurveda was not the preferred choice for most participants although a few reported taking it along with their regular allopathic medication as they felt that Ayurveda by itself would not be effective in treating them. They all spoke of the importance of eating a balanced diet, of exercising regularly, taking their medication as advised and of regular follow-up with a physician. To this end, most participants had modified their lifestyles, although to varying degrees. They reported cutting down on rice-based food items and sweets and exercising to the extent possible. While some indicated that they had no difficulty in changing their diet, others found it difficult. Similarly, regular exercise too posed a challenge with many indicating lack of time, poor motivation and complaints of body aches.

\section{Awareness about DR}

The findings revealed a mixed picture regarding awareness about DR. For most, it was not a familiar term while a few were aware of it and of the need to undergo regular retinal screening. The understanding that diabetes could affect their eyes and that their vision could be impaired had been gleaned through interactions with doctors, other health staff they came into contact with and through posters on diabetes on display in the hospitals they had been to. Issues about the potential threat to their eyes on account of diabetes were often reiterated during these visits. Participants were more familiar with other eye problems like glaucoma and cataract but for the most part remained unaware of the details and symptom manifestations of DR, and of possible preventive measures that needed to be taken to protect their eyes from DR. Only a couple of participants indicated that they had been informed about possible risks to their eyes on account of diabetes or of the precautions they needed to take to protect their eyes. The few who had heard about DR described it as a condition where the 'nerve would get affected'. They spoke of the importance of eye care, of regular eye check-up and the importance of keeping their blood sugar level under control as ways and means of protecting their eyes. Such participants were generally better educated, tended to discuss their health issues with their doctors and were more compliant.

\section{Barriers to DR screening}

Among those unfamiliar or less aware about DR, several issues emerged which acted as barriers to seeking eye care. A typical one related to consulting a doctor only if there was pain or some discomfort in the eye. In the absence of any symptoms it was deemed unnecessary to seek such eye care. Participants also feared that undergoing eye screenings and tests could result in more medicines being prescribed. Apart from concerns about cost and managing the dosage, they believed that these medicines meant more chemicals being ingested which was perceived as harmful as it contributed to excessive 'heat'. Others complained about doctors being too busy and of not having the time to talk to patients about all the dos and don'ts regarding diabetic eye care. If the doctor appeared too curt or busy, patients felt dissatisfied. But patients who indicated that they were doing well were generally satisfied with the care received and also tended to be more adherent to the doctor's advice. Other issues involved the logistics of travelling to the health facility, costs associated with undergoing the tests, not having the time to go for a check-up on account of work and family commitments. Some female participants spoke of not having anyone to accompany them to the health facility and almost all described the long hours they had to spend in the hospital to undergo these tests as major deterrents. Lastly, a sense of complacency and a lack of motivation were also cited as reasons for participants failing to seek regular care. In this context, one suggestion was for the hospital to send regular reminders to patients in the form 
of phone calls or phone messages informing patients that they were due for a check-up and encouraging them to visit the hospital. The above-mentioned patient's perspectives are summarised in online supplemental table 2.

\section{HCP perspectives}

Recognising and living with diabetes

The HCPs believed that people were largely aware about diabetes, referred to it as 'sugar disease' and understood that it required them to control their diet, restrict sweet intake and exercise regularly. Greater visibility of the disease was attributed to its high prevalence and widespread media coverage which had contributed to considerable awareness among people. Patients who were educated were more aware and had access to a wide range of information sources, like the internet, medical literature and health-related broadcasts on radio and television. These patients also sought further clarifications from their doctors and even questioned them when in doubt. On the other end of the spectrum were the poorer, often less educated patients who were not so knowledgeable about the disease and who also tended to be less compliant. The HCPs also spoke about issues concerning monitoring and controlling blood sugar levels which according to them was often not adequately maintained or even understood by patients. Thus, patients were generally aware about the disease, but the extent and depth of knowledge of what exactly they were up against varied considerably. In this context, the importance of proper counselling that would educate patients about diabetes and motivate them to attend regular reviews to the hospital was stressed. A few HCPs suggested the importance of exposing patients to all the possible diabetes-related complications by showing them pictures or getting them to meet other patients. This would impress on patients the seriousness of the problem.

\section{Care-seeking practices}

In terms of issues related to care seeking, all HCPs uniformly said that in addition to telling patients about the disease, its symptom manifestations and its management strategies, they reiterated the need to undergo periodic blood tests to monitor their blood sugar level and ensure that they kept it under control. The importance of seeking care from a diabetologist was also stressed as these doctors had the expertise to guide and appropriately advise patients. Further, they advised that as the disease could affect any of their internal organs and was basically a 'silent killer', it was imperative that patients underwent regular check-up. Usually the information was conveyed to patients often with the use of printed pamphlets every time the patient visited the health facility. One HCP, an ophthalmologist, declared that he typically advised his patients with diabetes to undergo an HbAlc in addition to fasting and postprandial blood tests. He also advised them to undergo kidney and liver function tests and check their cholesterol and blood pressure as their diabetes could get exacerbated by other prevailing comorbidities. The HCPs thus spoke of following a fairly structured protocol which also entailed constantly emphasising the importance of lifestyle modifications as being critical to maintaining health. Use of posters and slogans educating people about the disease and emphasising the importance of regular care was also highlighted. A barrier to proper care highlighted by the HCPs was the availability of a plethora of information on social media sites about diabetes and related health problems. Most of this information was either inadequate or incorrect and those who tended to follow it did so at great cost to themselves. Another HCP, a dietician, spoke of gearing the information to the patient's level of understanding, breaking it down to simple dos and don'ts which she felt was easier for the patient to follow. This was feasible for her to do as she had more time with the patient unlike the doctors. Patient's motivation levels and presence of good family support were also seen as aids to good compliance.

\section{Awareness about DR and barrier to DR screening}

The general opinion among the HCPs was that awareness about DR was still poor in patients with very few having heard of it. They accepted that patients knew that diabetes could affect the eyes, were familiar with cataract, but for the most remained unaware of DR. One HCP, an ophthalmologist, described two types of patients with diabetes: (1) those who remained unaware that the disease could affect their eyes and blamed their doctors for failing to educate them adequately and (2) those who despite being asked to attend a retinal screening failed to do so as they did not suffer any symptoms. This silent and quiet progression of DR where patients largely experienced no symptoms resulted in patients not perceiving the need to seek care thereby seriously compromising their vision. In this context, one HCP said that many Indian patients normally come for a check-up when there is an 'acute crisis or acute problem' and unless and until they experienced some difficulties, they usually did not seek care. Cost and lack of time were other issues particularly for poorer persons and those working on daily wages resulting in delays in seeking care. Explaining the nuances of the disease to such patients who often tended to have low literacy was found to be quite a challenge. Lack of motivation; financial problems; absence of good family/ social support in terms of someone to accompany them to the hospital; and slow improvement in vision following initiation of treatment acted as deterrents to continued care seeking. Patients also tended to be complacent if their blood sugar levels were under control, little realising that the longer the duration of diabetes, the greater was their risk of developing DR. The above-mentioned HCP perspectives are mentioned in online supplemental table 3.

\section{DISCUSSION}

This qualitative study has provided important insights into barriers to regular screening for DR from the perspectives of patients and providers across four themes: (1) recognising and living with diabetes, (2) care-seeking 
practices, (3) awareness about DR, and (4) barriers to DR screening. Patients were largely aware of diabetes, its symptoms and importance of diet and medication management and of exercise which were also endorsed by the HCPs. It was also evident that the management of this disease imposed a tremendous burden on both HCPs and patients alike. For providers, communicating the complexities of the disease in words that patients could understand and keeping them motivated to ensure good compliance proved challenging. For patients, the burden of constantly having to follow a healthy lifestyle, being systematic in seeking care combined with a lack of depth in their understanding of the disease contributed to them feeling overwhelmed and frustrated, even depressed.

A study by $\mathrm{Li}$ et $a t^{22}$ highlighted the importance of addressing depression in people with diabetes and recommended the need to motivate patients to exercise and follow a healthy lifestyle. The fact that diabetes can affect the eyes was reported by most although awareness about DR was poor, a fact confirmed by the HCPs. Poor understanding of DR has also been reported by patients in other studies where they expressed having no knowledge about the possibility of becoming blind on account of diabetes. ${ }^{23}{ }^{24}$ In another study, ${ }^{25}$ despite most participants being aware about the need to undergo eye examinations, there was limited understanding about retinopathy and about the rationale behind the recommendation.

Apart from feeling overwhelmed and frustrated with the care routines, the prospect of having to spend more than half a day at the hospital, as health facilities here are mostly very crowded, was another major deterrent to care seeking for DR. Patients therefore tended to delay seeking care and clung to the belief that as they were feeling alright there was no requirement to go to the hospital. This absence of symptoms which created a sense of complacency among patients, which was also a theme reported in the systematic review by Graham-Rowe et $a l,{ }^{26}$ emerged as a major deterrent to undergoing eye screening for DR in our study. Patients questioned the need to undergo eye tests which were usually tedious and required them to spend long hours in the hospital. Further, they feared having to take more medications that they thought were unnecessary as they experienced no symptoms. Besides, it meant ingesting more chemicals contributing to excessive heat in their bodies. This cultural belief in the concept of excessive heat and cold attributed to both modern medicines and foods dates back to the Charaka Samhita, a Sanskrit text on Ayurveda (Indian traditional medicine), and has deep roots in the minds of people. ${ }^{27} 28$ The HCPs agreed that the silent progression of DR was a deterrent to early care seeking and spoke of difficulties they faced in getting patients to understand the importance of early and regular eye screening and testing.

Thus, strategies that enhance patient understanding of the disease are needed. In this context, a study carried out by Trento et $a l^{29}$ showed that patients who participated in group sessions understood DR better. The effectiveness of peer support as a method of increasing uptake of DR screening is a concept that is to be tested in a proposed trial in Kenya. ${ }^{30}$ Such studies will help prove whether being part of a peer groups enhances long-term support to group members thereby acting as an incentive to remain compliant. In our setting, educating patients about diabetes is mostly didactic, and happens during the brief consultation sessions with the doctors and subsequently during their interaction with other healthcare staff. HCPs have found communication packages like conversation maps, which are interactive illustrations, helpful to educate patients with DM about the importance of self-care, as a means to prevent/ delay the onset of related complications. ${ }^{31}$ Telescreening has been found to be promising in terms of improving compliance apart from being cost-effective ${ }^{32}$ for a rural population. Improving awareness about diabetes and its complications among community health workers such as the accredited social health activists in India, who have worked well for other health issues like maternal and child health and infectious diseases like HIV ${ }^{33}{ }^{34}$ may be a way forward. Future research could test the application of such strategies.

Another important point that emerged was the nature of the doctor-patient interactions. Many patients were critical of doctors who they felt did not explain adequately or were always in a rush. Some spoke of the manner in which doctors communicated to them leaving them feeling threatened and frightened, and therefore more likely to switch to another doctor. They felt confused when meeting different doctors on account of their conflicting opinions. Patients looked to their HCPs for support and encouragement that was often not forthcoming on account of their busy schedules. Doctors are often hard pressed for time which compromises their ability to spend quality time with patients, a feature that was highlighted by many in our study. The HCPs felt that despite repeatedly talking to patients about the disease and its complications, many patients did not appreciate the importance of regularly monitoring and maintaining their blood sugar levels and attending for eye screening. They expressed difficulties communicating to less literate persons who were often shown to be less compliant. The need of patients for HCPs to be more approachable has been expressed by patients in other studies as well. Peel $e t$ $a l^{35}$ reported that participants in their study wanted more support and information from their HCPs and felt frustrated as many of their concerns had not been answered. Maddigan $e t a l^{36}$ described the value of good patientprovider relationships as contributing to good exercise adherence thereby improving quality of life.

It is apparent that HCPs play a pivotal role in promoting understanding of the disease given the almost reverential position they occupy in our culture. At the same time, patients' expectations of doctors are also very high and if they feel that they are not improving to their satisfaction, an element of distrust and unhappiness tends to creep in which in turn colours their opinions. Our study 
findings further showed that there is a gap between what is conveyed to patients by the HCPs, and how much of that is actually understood by them. Perhaps the strategy of 'one size fits all' where standard information is provided to all patients needs to be addressed in the form of healthcare awareness and education by counsellors. ${ }^{3738}$ Due consideration to a patient's understanding capacity, self-efficacy, attitudes and health beliefs ${ }^{39}$ which exert an influence on their lifestyle management would aid HCPs to improve their communication skills and enhance patient understanding. Reducing the burden on doctors, perhaps by building a comprehensive diabetic care team comprising trained personnel, some of whom could take on the role of educating and counselling patients while doctors could focus on care delivery, could be a possible strategy. Educating people about the skill sets and roles of each member of the team will also be essential to promote acceptance. Such an integrated approach where care of diabetes and its complications are available under one roof, literally a 'one-stop shop', indicative of a paradigm shift compared with what is currently practised (online supplemental file 1), seems the most logical way going forward.

This qualitative study by exploring perspectives of both patients and HCPs has provided useful insights which have the potential to guide future intervention development. The study could have benefited from interviews with family members, who play an important role in decision-making for care seeking and in providing support to patients. Inclusion of HCPs from smaller eye clinics would have provided additional perspectives further enhancing understanding of the phenomena. A larger patient sample representative of a wider patient demographic could perhaps have brought in more perspectives.

\section{CONCLUSION}

Living with and managing diabetes is a lifelong process, one that can prove overwhelming to an unprepared patient. It is therefore imperative that steps to ensure good patient compliance be prioritised. Enhancing patient understanding through healthy and friendly doctorpatient interactions and use of an integrated treatment approach including education by counsellors, setting up patient support groups, telescreening approaches and use of conversation maps are some strategies that may prove more effective in enhancing compliance for DR care.

Acknowledgements We would like to thank Dr Rajalakshmi and the staff of $\mathrm{Dr}$ Mohan's Diabetes Specialities Centre for their support in conducting interviews with healthcare providers. We thank the social workers for helping contact patients.

Contributors RR and SK contributed to the conception and design of the study. SK wrote the main manuscript text. GK and SV assisted with data collection and statistical analyses. RR, SK, GK and SV drafted the work and SP, PR and SS substantively revised it. All authors reviewed the manuscript.

Funding This study was supported by the Lions Club International Foundation (SFP2050/UND).

Competing interests None declared.
Patient consent for publication Obtained.

Ethics approval The study was approved by the Institutional Review Board (Ethics Committee) of Vision Research Foundation and written consent was obtained from the patients as per the Declaration of Helsinki.

Provenance and peer review Not commissioned; externally peer reviewed.

Data availability statement Data are available upon reasonable request.

Supplemental material This content has been supplied by the author(s). It has not been vetted by BMJ Publishing Group Limited (BMJ) and may not have been peer-reviewed. Any opinions or recommendations discussed are solely those of the author(s) and are not endorsed by BMJ. BMJ disclaims all liability and responsibility arising from any reliance placed on the content. Where the content includes any translated material, BMJ does not warrant the accuracy and reliability of the translations (including but not limited to local regulations, clinical guidelines, terminology, drug names and drug dosages), and is not responsible for any error and/or omissions arising from translation and adaptation or otherwise.

Open access This is an open access article distributed in accordance with the Creative Commons Attribution Non Commercial (CC BY-NC 4.0) license, which permits others to distribute, remix, adapt, build upon this work non-commercially, and license their derivative works on different terms, provided the original work is properly cited, appropriate credit is given, any changes made indicated, and the use is non-commercial. See: http://creativecommons.org/licenses/by-nc/4.0/.

ORCID iD

Rajiv Raman http://orcid.org/0000-0001-5842-0233

\section{REFERENCES}

1 Thomas RL, Halim S, Gurudas S, et al. IDF diabetes atlas: a review of studies utilising retinal photography on the global prevalence of diabetes related retinopathy between 2015 and 2018. Diabetes Res Clin Pract 2019;157:107840.

2 Yau JWY, Rogers SL, Kawasaki R, et al. Global prevalence and major risk factors of diabetic retinopathy. Diabetes Care 2012;35:556-64.

3 Flaxman SR, Bourne RRA, Resnikoff S, et al. Global causes of blindness and distance vision impairment 1990-2020: a systematic review and meta-analysis. Lancet Glob Health 2017;5:e1221-34.

4 King H, Aubert RE, Herman WH. Global burden of diabetes, 19952025: prevalence, numerical estimates, and projections. Diabetes Care 1998;21:1414-31.

5 Raman R, Gella L, Srinivasan S, et al. Diabetic retinopathy: an epidemic at home and around the world. Indian J Ophthalmol 2016;64:69-75.

6 Gilbert C, Gordon I, Mukherjee CR, et al. Guidelines for the prevention and management of diabetic retinopathy and diabetic eye disease in India: a synopsis. Indian J Ophthalmol 2020;68:S63.

7 Ramasamy K, Raman R, Tandon M. Current state of care for diabetic retinopathy in India. Curr Diab Rep 2013;13:460-8.

8 Shukla R, Gudlavalleti MVS, Bandyopadhyay S, et al. Perception of care and barriers to treatment in individuals with diabetic retinopathy in India: 11-city 9-state study. Indian J Endocrinol Metab 2016;20:S33-41.

9 Lingam S, Rani PK, Sheeladevi S, et al. Knowledge, attitude and practices on diabetes, hypertension and diabetic retinopathy and the factors that motivate screening for diabetes and diabetic retinopathy in a pyramidal model of eye health care. Rural Remote Health 2018;18:4304.

10 Coney JM. Addressing unmet needs in diabetic retinopathy. Am J Manag Care 2019;25:S311-6.

11 Vengadesan N, Ahmad M, Sindal MD, et al. Delayed follow-up in patients with diabetic retinopathy in South India: social factors and impact on disease progression. Indian J Ophthalmol 2017;65:376-84.

12 Rohan TE, Frost CD, Wald NJ. Prevention of blindness by screening for diabetic retinopathy: a quantitative assessment. BMJ 1989;299:1198-201.

13 Namperumalsamy P, Nirmalan PK, Ramasamy K. Developing a screening program to detect sight-threatening diabetic retinopathy in South India. Diabetes Care 2003;26:1831-5.

14 KashimRM, NewtonP, OjoO, et al. Diabetic retinopathy screening: A systematic review on patients' non-attendance. Int J Environ Res Public Health 2018;15:157.

15 Lu Y, Serpas L, Genter P, et al. Divergent perceptions of barriers to diabetic retinopathy screening among patients and care providers, Los Angeles, California, 2014-2015. Prev Chronic Dis 2016;13:160193. 
16 Piyasena MMPN, Murthy GVS, Yip JLY, et al. A qualitative study on barriers and enablers to uptake of diabetic retinopathy screening by people with diabetes in the Western Province of Sri Lanka. Trop Med Health 2019:47:34.

17 Peng P-H, Laditka SB, Lin H-S, et al. Factors associated with retinal screening among patients with diabetes in Taiwan. Taiwan $J$ Ophthalmol 2019;9:185-93.

18 Raman R, Rani PK, Reddi Rachepalle S, et al. Prevalence of diabetic retinopathy in India: Sankara Nethralaya diabetic retinopathy epidemiology and molecular genetics study report 2. Ophthalmology 2009;116:311-8.

19 Guest G, Bunce A, Johnson L. How many interviews are enough? an experiment with data saturation and variability. Field Methods 2006;18:59-82.

20 Ritchie J, Lewis J, Nicholls CM, et al. Qualitative research practice: a guide for social science students and researchers. sage, 2013.

21 Gale NK, Heath G, Cameron E, et al. Using the framework method for the analysis of qualitative data in multi-disciplinary health research. BMC Med Res Methodol 2013;13:1-8.

22 Li D, Inouye J, Davis J, et al. Associations between psychosocial and physiological factors and diabetes health indicators in Asian and Pacific Islander adults with type 2 diabetes. Nurs Res Pract 2013;2013:1-7.

23 Pasagian-Macaulay A, Basch CE, Zybert P, et al. Ophthalmic knowledge and beliefs among women with diabetes. Diabetes Educ 1997;23:433-7.

24 Lewis K, Patel D, Yorston D, et al. A qualitative study in the United Kingdom of factors influencing attendance by patients with diabetes at ophthalmic outpatient clinics. Ophthalmic Epidemiol 2007;14:375-80.

25 Hartnett ME, Key IJ, Loyacano NM, et al. Perceived barriers to diabetic eye care: qualitative study of patients and physicians. Arch Ophthalmol 2005;123:387-91.

26 Graham-Rowe E, Lorencatto F, Lawrenson JG, et al. Barriers to and enablers of diabetic retinopathy screening attendance: a systematic review of published and grey literature. Diabet Med 2018;35:1308-19.

27 Selin H. A history of Indian medical literature, 2002.

28 Satyavati GV. The legacy of Caraka, 2003.
29 Trento M, Bajardi M, Borgo E, et al. Perceptions of diabetic retinopathy and screening procedures among diabetic people. Diabet Med 2002;19:810-3.

30 Mwangi N, Ng'ang'a M, Gakuo E, et al. Effectiveness of peer support to increase uptake of retinal examination for diabetic retinopathy: study protocol for the DURE pragmatic cluster randomized clinical trial in Kirinyaga, Kenya. BMC Public Health 2018;18:871.

31 Carvalho SLde, Ferreira MA, Medeiros JMP, et al. Conversation map: an educational strategy in the care of elderly people with diabetes mellitus. Rev Bras Enferm 2018;71 Suppl 2:925-9.

32 Rachapelle S, Legood R, Alavi Y, et al. The cost-utility of telemedicine to screen for diabetic retinopathy in India. Ophthalmology 2013;120:566-73.

33 Lassi ZS, Bhutta ZA. Community-Based intervention packages for reducing maternal and neonatal morbidity and mortality and improving neonatal outcomes. Cochrane Database Syst Rev 2015:Art. No.: CD007754.

34 Busza J, Dauya E, Bandason T, et al. The role of community health workers in improving HIV treatment outcomes in children: lessons learned from the ZENITH trial in Zimbabwe. Health Policy Plan 2018;33:328-34.

35 Peel E, Parry O, Douglas M, et al. Diagnosis of type 2 diabetes: a qualitative analysis of patients'emotional reactions and views about information provision. Patient Educ Couns 2004;53:269-75.

36 Maddigan SL, Majumdar SR, Johnson JA. Understanding the complex associations between patient-provider relationships, self-care behaviours, and health-related quality of life in type 2 diabetes: a structural equation modeling approach. Qual Life Res 2005; 14:1489-500.

37 Malathy R, Narmadha M, Ramesh S, et al. Effect of a diabetes counseling programme on knowledge, attitude and practice among diabetic patients in Erode district of South India. $J$ Young Pharm 2011;3:65-72.

38 Adepu R, Rasheed A, Nagavi BG. Effect of patient counseling on quality of life in type-2 diabetes mellitus patients in two selected South Indian community pharmacies: a study. Indian J Pharm Sci 2007;69:519.

39 Peel E, Douglas M, Lawton J. Self monitoring of blood glucose in type 2 diabetes: longitudinal qualitative study of patients' perspectives. BMJ 2007;335:493. 